\title{
Diagnostic performance of superb microvascular imaging combined with shear-wave elastography in distinguishing invasive ductal carcinoma molecular subtypes
}

\author{
Fengjiao Chen, Hui Jing, Haitao Shang, Haoyan Tan, Haobo Yang, Mengqiong Kong, Wen Cheng
}

Harbin Medical University Cancer Hospital, Nangang District, Harbin, China

Submitted: 23 August 2021; Accepted: 7 January 2022

Online publication: 13 January 2022

Arch Med Sci

DOI: https://doi.org/10.5114/aoms/145596

Copyright @ 2022 Termedia \& Banach

\section{Abstract}

Introduction: The aim of the study was to explore the diagnostic value of combining superb microvascular imaging (SMI), shear-wave elastography (SWE), and the Breast Imaging Reporting and Data System (BI-RADS) to distinguish different molecular subtypes of invasive ductal carcinoma (IDC).

Material and methods: A total of 239 surgically confirmed IDC masses in 201 patients underwent conventional ultrasound, SMI, and SWE examination; information such as echo pattern, posterior features, margins, SMI pixels, and hardness of the masses was recorded. According to the St. Gallen standard, breast masses were classified as Luminal A, Luminal B, HER2 overexpression, and triple-negative subtype. We further explored the differences between different molecular subtypes of IDC.

Results: Luminal A subtype had the following characteristics: low histologic grade, posterior acoustic shadowing $(p=0.019)$, spiculated margins $(p<0.001)$, and relatively soft. Luminal B subtype was characterized by low histological grade $(p<0.0001)$, posterior acoustic shadowing or indifference, and indistinct margins. HER2 overexpression breast cancers were characterized by high histological grade, enhanced posterior acoustics or indifference, calcifications $(p=0.005)$, spiculated or indistinct margins, vascularity $(p=0.005)$, and relative stiffness. Triple-negative breast cancers had the characteristics of high histological grade, posterior echogenic enhancement, lack of calcifications, circumscribed or microlobulated margins, low blood flow signals, and stiff tissue $(p=0.013)$.

Conclusions: Our study demonstrated the significant differences and trends among the four IDC subtypes by the combined application of SMI, SWE, and BI-RADS lexicon, which are of great significance for early diagnosis, selection of treatment methods, and evaluation of prognosis of IDC.

Key words: superb microvascular imaging, shear-wave elastography, invasive ductal carcinoma, molecular subtype.

\section{Introduction}

Five years of epidemiological data show that approximately $11 \%$ of breast cancers around the world occurred in China, and the incidence has increased rapidly in recent decades [1]. Breast cancer is a highly heterogeneous disease, and invasive ductal carcinoma (IDC) is the most common type and is classified into Luminal A (LA), Luminal B (LB), hu-

\author{
Corresponding authors: \\ Mengqiong Kong, \\ Wen Cheng \\ Harbin Medical \\ University Cancer \\ Hospital \\ 150 Haping Road \\ Nangang District \\ Harbin, 150080 \\ China \\ Phone: +8613313677182 \\ Fax: +85 0451-85718395 \\ E-mail: \\ kongmengqiong@163.com, \\ hrbchengwen@163.com
}


man epidermal growth factor receptor 2-positive (HER2+) overexpression, and triple-negative (TN) subtypes [2]. Different molecular subtypes of breast cancer have distinct biological characteristics, treatment methods, and clinical prognoses [2-4]. Therefore, effective differentiation of molecular subtypes of breast cancer is beneficial for clinical diagnosis, treatment, and prognosis evaluation. Immunohistochemistry is used for breast cancer molecular typing [3]. However, the immunohistochemistry approach relies on obtaining pathological specimens, which is invasive, resulting in a delay in establishing the diagnosis. Therefore, imaging methods play a significant role in the diagnosis of breast diseases.

With the characteristics of non-invasiveness, being real-time dynamic, and having high diagnostic sensitivity and specificity, ultrasound has become a simplified breast disease diagnostic method $[5,6]$. Angiogenesis promotes tumour growth, invasion, and distant metastasis [7]. Based on the close relationship between microvascularity and malignancy, an emerging Doppler ultrasound method, called superb microvascular imaging (SMI), has been used to visualize microvessels. This technique relies on an intelligent algorithm that can effectively distinguish low-velocity blood flows from motion artefacts so that it can display low-velocity blood flow signals in microvessels [8, 9]. Another potential feature used to distinguish breast masses is tissue stiffness. In this context, shear-wave elastography (SWE) provides quantitative and reproducible hardness information about breast masses, which is at least as diagnostically effective as the greyscale ultrasound with the Breast Imaging Reporting and Data System (BI-RADS) classification [10, 11].

However, to the best of our knowledge, there are few reports about the use of SMI, SWE, and BIRADS lexicon to distinguish molecular subtypes of IDC. Therefore, this study investigated the efficacy of the combined application of SMI in exploring blood vessels and SWE in measuring tissue stiffness to distinguish the molecular subtypes of IDC.

\section{Material and methods}

\section{Patients}

This retrospective study was approved by the Ethics Committee of Harbin Medical University Cancer Hospital, and informed consent for secondary use of medical history data was obtained from all participants. Data were collected from 300 patients with breast masses who underwent surgery in Harbin Medical University Cancer Hospital between December 2018 and May 2019. Sixty-one of the 300 patients with benign breast masses, without molecular classifications, distant metas- tasis, or pregnancy, were excluded from the study. To obtain better SWE and SMI ultrasound images, 38 of the 239 patients with tumours larger than $50 \mathrm{~mm}$ in diameter and deeper than $40 \mathrm{~mm}$ were excluded. A total of 201 IDC patients with standard ultrasound images and complete clinicopathological data were included in the study.

\section{Ultrasound examinations}

Age and reason for visiting the doctor of all patients were recorded. Ultrasonography was conducted using a Canon Aplio i900 (Canon Medical System Corporation) equipped with a 5-18 MHz high-frequency line array probe. An experienced radiologist performed the conventional ultrasound, SMI, and SWE examinations on all patients with at least two orthogonal views. Traditional ultrasound examination was performed for all patients, and we recorded the following information: position of the lesion (left or right), lesion size, maximum depth (vertical distance from the skin to the lesion bottom), shape (round/oval or irregular), orientation (parallel or not parallel), echogenicity (hypoechoic or complex cystic and solid), posterior features (no posterior features, shadowing, enhancement, combined pattern), presence of calcifications and margins (indistinct, circumscribed, angular, spiculated, microlobulated). All lesions were rated according to the latest ultrasound BI-RADS of the American College of Radiology [12].

Then we performed SMI for the breast lesions, and there was little pressure applied on the probe to avoid blood vessel collapse. The speed range of the SMI was adapted to $1-2 \mathrm{~cm} / \mathrm{s}$. Static and dynamic images of the patients were stored in the machine. The images were then evaluated by two doctors who are well experienced with breast ultrasound and had been trained in SMI; disagreement was resolved by discussion until they reached a common decision. We characterized the entire lesion as the region of interest (ROI) and assessed the ROI with Adler's classification, which is used to classify blood flow. Vascularity was graded from 0 to 3 depending on the number of vessels. Grade 0 means no blood flow. Minimal (Grade 1) means one or two pixels containing flow (usually less than $0.1 \mathrm{~cm}$ in diameter). When a certain number of small vessels and/or the main vessel was visible, it was defined as moderate (Grade 2). Marked (Grade 3) was defined when four or more blood vessels were visible (Figure 1). Meanwhile, we measured SMI pixels, $\mathrm{ROI}$ pixels, $\mathrm{SMI}$ area, $\mathrm{ROI}$ area, and the ratio of SMI pixels to ROI pixels (Figure $2 \mathrm{~A}$ ).

The same radiologist performed SWE on the breast lesions without compression. After locating the breast mass on the section with the largest diameter, the radiologist placed it at the centre of 

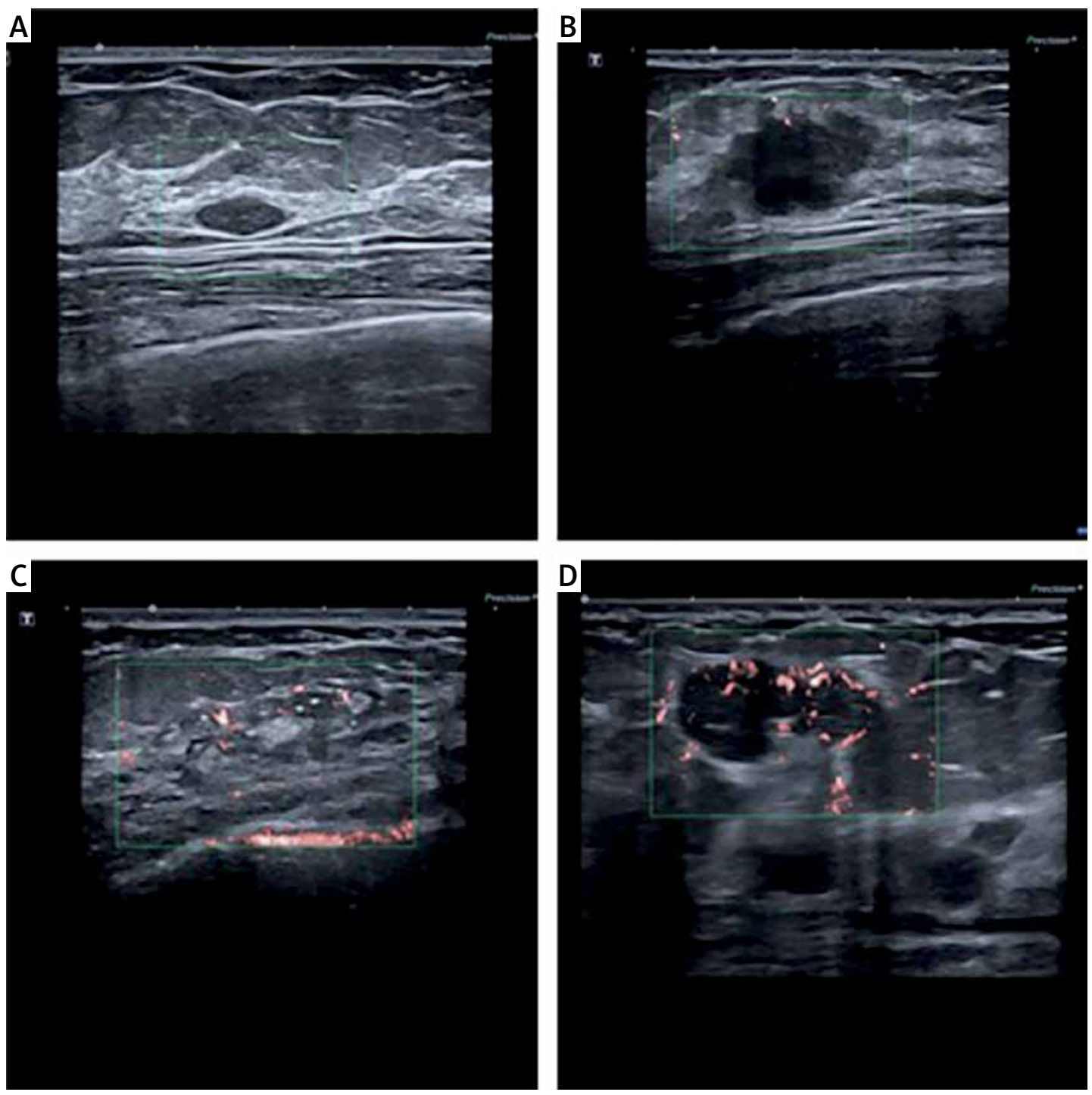

Figure 1. Evaluation of blood build-up in breast masses was graded according to Adler's classification: A-D - represented grade 0 to 3
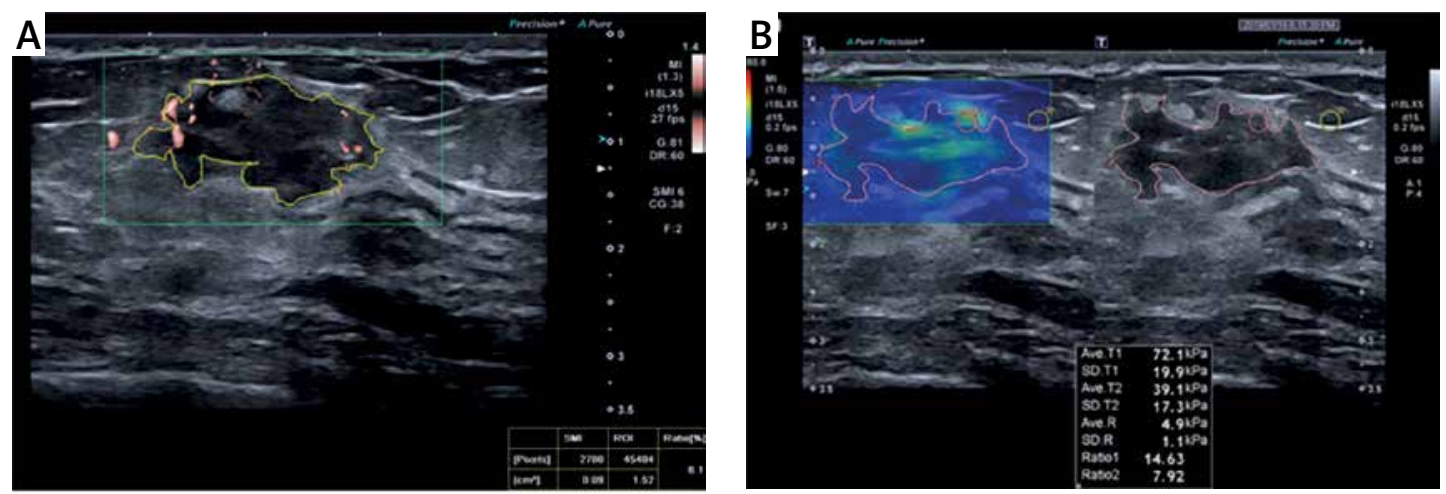

Figure 2. A - We can measure SMI pixels, ROI pixels, SMI area, ROI area and the ratio of SMI pixels to ROI pixels with the SMI measurement software; B - We obtained the average of T1 (Ave.T1), the standard deviation of T1 (SD.T1); the average of T2 (Ave.T2), the standard deviation of T2 (SD.T2); the average value (Ave.R) and standard deviation (SD.R) of region R; the ratio of Ave.T1 to Ave.R (Ratio1) and the ratio of Ave.T2 to Ave.R (Ratio2) using the elasticity measurement tool 
the elasticity box, and the SWE image was stored after a few seconds of immobilization to allow the image to stabilize. For SWE, the colour image representing hardness was overlaid on the conventional B-mode image, ranging from dark blue, representing the softest, to red, representing the hardest $(0-180 \mathrm{kPa})$, with the $\mathrm{B}$-mode image alone shown just next to it. The same two doctors who evaluated SMI images conducted elastic quantitative measurements. The circular ROI $2 \mathrm{~mm}$ in diameter placed at the stiffest part of the mass was labelled as ROI-T1, manual tracking of the boundary of the tumour was labelled as ROI-T2, and the circular ROI $2 \mathrm{~mm}$ in diameter placed at the normal mammary gland was labelled ROI-R. Then, we obtained the average (Ave.T1) and standard deviation (SD.T1) of T1, the average (Ave.T2) and standard deviation (SD.T2) of T2, the average (Ave.R) and standard deviation (SD.R) of region R, the ratio of Ave.T1 to Ave.R (Ratio1) and the ratio of Ave.T2 to Ave.R (Ratio2) (Figure 2 B).

\section{Histopathological characteristics and tissue specimens}

We determined the pathological type, histological grade, and lymph node metastasis of the tumours according to postoperative pathological results. The histological grade of the tumour was classified as grade 1 (well-differentiated), grade 2 (moderately differentiated), or grade 3 (poorly differentiated) [13]. We considered grades 1 and 2 to be low and grade 3 to be high. The histopathological characteristics included are oestrogen receptor (ER), progesterone receptor (PR), HER2, and Ki-67 indicators. Immunohistochemistry staining of $4 \mu \mathrm{m}$ sections of formalin-fixed paraffin-embedded tissue was performed with anti-ER (clone SP1, Ventana), anti-PR (clone 1E2, Ventana), anti-HER2 (clone 4B5, Ventana), anti-Ki-67 (clone 30-9, Ventana) rabbit monoclonal primary antibodies. Universal secondary antibody was applied for 15 min. Diaminobenzidine was used as chromogens and slides were counterstained with haematoxylin before mounting. Leica Microsystems equipment (CMS GmbH, Wetzlar, Germany) was used for evaluation. ER and PR expression was considered positive when nuclear staining was greater than $1 \%$ and $20 \%$, respectively $[2,14,15]$. HER2 positive was defined as $3+$, and fluorescence in situ hybridization was performed in patients with 2+ for positive diagnosis [16]. The proportion of Ki-67-stained cells among all tumour cells was considered as Ki-67 status.

Intrinsic molecular subtypes were classified according to the St. Gallen International Expert Consensus on the Primary Therapy of Early Breast Cancer 2013 [2] as follows: (1) the LA subtype was "ER and PR positive, HER2 negative, and Ki-
67 low (< 14\%)"; (2) the LB subtype was divided into HER2 + subtype (ER-positive, HER2 overexpressed or amplified, any Ki-67, and any PR) and HER2- subtype (ER-positive, HER2 negative, and at least one of the following: Ki-67 $\geq 14 \%$, PR negative or low, and high recurrence risk based on multi-gene-expression assay); (3) the HER2+ subtype was "HER2 overexpressed or amplified, and ER and PR absent"; and (4) the triple-negative breast cancer (TNBC) subtype was "ER and PR absent, and HER2 negative".

\section{Statistical analysis}

Normally distributed data are presented as mean \pm standard deviation and abnormally distributed data as median (interquartile range). Differences were assessed using analysis of variance, Student's $t$-test, Kruskal-Wallis $H$ test, or Wilcoxon rank-sum test. Multiple comparisons were performed using the SNK test or Nemenyi test. Frequency (percentage) is used for the statistical description of qualitative data. Categorical data were compared by the $\chi^{2}$ test or Fisher's exact test, and an ordinal categorical variable was compared using the Kruskal-Wallis $H$ test or Wilcoxon rank-sum test. All statistical analyses were performed using SAS 9.1.3 software, and statistical significance was set at $p<0.05$.

\section{Results}

A total of 239 breast masses were removed from the 201 patients with IDC (mean age: 51 years, range: $27-78$ years). LA subtype accounted for $33.89 \%(n=81)$; LB, $46.03 \%(n=110)$; HER $2+$, $10.88 \%(n=26)$; and TNBC, 9.20\% $(n=22)$. From the 110 LB subtypes, LB (HER2+) and LB (HER2-) accounted for $25.45 \%(n=28)$ and $74.55 \%(n=82)$, respectively. The clinicopathological data and sonographic parameters of the four IDC molecular subtypes and two LB subtypes are shown in Tables I-IV.

\section{Clinicopathological comparisons among the four molecular subtypes of IDC}

Perimenopausal women were at higher risk for IDC, especially those aged 40-49 years. There was no statistically significant difference in clinical symptoms among the four groups. Our results indicated that there was a statistical difference in the histological grade among the four subtypes, with a high grade more common in HER2+ (57.69\%) and TNBC (72.73\%) subtypes and less common in LA (14.82\%) and LB (20.91\%) subtypes. The HER2+ group had lower frequency of grade 1 than that in LA $(p=0.00038)$ and LB ( $p=0.02163)$, and the TNBC subtype had lower frequency of grade 1 than that in LA $(p=0.000021)$ 
Table I. Comparisons of clinicopathological and sonographic characteristics among the four molecular subtypes of invasive ductal carcinoma

\begin{tabular}{|c|c|c|c|c|c|c|}
\hline Variables & LA $(n=81)$ & LB $(n=110)$ & HER2 $+(n=26)$ & $\mathrm{TN}(n=22)$ & $\chi^{2} / F$ value & $P$-value \\
\hline Age [years] & $51.60 \pm 10.37$ & $49.68 \pm 9.46$ & $50.50 \pm 6.960$ & $53.44 \pm 9.34$ & 0.998 & 0.395 \\
\hline Symptoms: & & & & & & 0.510 \\
\hline Yes & $72(88.89)$ & $90(81.82)$ & $21(80.77)$ & $18(81.82)$ & & \\
\hline No & $9(11.11)$ & $20(18.18)$ & $5(19.23)$ & 4 (18.18) & & \\
\hline Histological grade: & & & & & 40.166 & $<0.0001$ \\
\hline 1 & $20(24.69)$ & $11(10.00)$ & $1(3.85)^{\star \#}$ & $1(4.54)^{\star \#}$ & & \\
\hline 2 & $49(60.49)$ & 76 (69.09) & $10(38.46)$ & $5(22.73)$ & & \\
\hline 3 & $12(14.82)$ & $23(20.91)$ & $15(57.69)$ & $16(72.73)$ & & \\
\hline Lymph node metastasis: & & & & & 2.265 & 0.519 \\
\hline Yes & $30(37.04)$ & $39(35.45)$ & $6(23.08)$ & $6(27.27)$ & & \\
\hline No & $51(62.96)$ & $71(64.55)$ & $20(76.92)$ & $16(72.73)$ & & \\
\hline Position & & & & & 2.455 & 0.484 \\
\hline Left & $39(48.15)$ & $59(53.64)$ & $17(65.38)$ & $11(50)$ & & \\
\hline Right & $42(51.85)$ & $51(46.36)$ & $9(34.62)$ & $11(50)$ & & \\
\hline Size $[\mathrm{mm}]$ & $\begin{array}{c}19.5(15.8- \\
28.2)\end{array}$ & $\begin{array}{c}19.9(14.4- \\
28.7)\end{array}$ & $\begin{array}{c}27.0(13.5- \\
31.4)\end{array}$ & $\begin{array}{c}21.4(11.3- \\
33.2)\end{array}$ & 1.650 & 0.648 \\
\hline Maximum depth [mm] & $\begin{array}{c}19.5 \\
(16-24)\end{array}$ & $\begin{array}{c}19.7 \\
(16.6-23.7)\end{array}$ & $\begin{array}{c}19.8 \\
(16.5-24.7)\end{array}$ & $\begin{array}{c}21.6 \\
(18.3-25.6)\end{array}$ & 1.549 & 0.671 \\
\hline Shape: & & & & & & 0.476 \\
\hline Round/oval & $6(7.41)$ & $12(10.91)$ & $2(7.69)$ & $4(18.18)$ & & \\
\hline Irregular & 75 (92.59) & $98(89.09)$ & $24(92.31)$ & $18(81.82)$ & & \\
\hline Orientation: & & & & & 12.568 & 0.006 \\
\hline Parallel & $49(60.49)$ & $77(70.00)$ & $24(92.31)$ & $19(86.36)$ & & \\
\hline Not parallel & $32(39.51)$ & $33(30.00)$ & $2(7.69)^{*}$ & $3(13.64)$ & & \\
\hline Echo pattern: & & & & & & $<0.001$ \\
\hline Hypoechoic & $57(70.37)$ & $101(91.82)^{\star}$ & $24(92.31)$ & $20(90.91)$ & & \\
\hline $\begin{array}{l}\text { Complex cystic } \\
\text { and solid }\end{array}$ & $24(29.63)$ & $9(8.18)$ & $2(7.69)$ & $2(9.09)$ & & \\
\hline Posterior features: & & & & & 19.787 & 0.019 \\
\hline No posterior features & $19(23.46)$ & $34(30.91)$ & $10(38.46)$ & $6(27.27)^{*}$ & & \\
\hline Shadowing & $45(55.55)$ & $43(39.09)$ & $6(23.08)$ & $4(18.18)$ & & \\
\hline Enhancement & $13(16.05)$ & $28(25.45)$ & $8(30.77)$ & $11(50)$ & & \\
\hline Combined pattern & $4(4.94)$ & $5(4.55)$ & $2(7.69)$ & $1(4.55)$ & & \\
\hline Calcification: & & & & & 13.034 & 0.005 \\
\hline Present & $43(53.09)$ & $34(30.91)^{\star}$ & $14(53.85)$ & $6(27.27)$ & & \\
\hline Absent & $38(46.91)$ & $76(69.09)$ & $12(46.15)$ & $16(72.73)$ & & \\
\hline Margin: & & & & & & $<0.001$ \\
\hline Indistinct & $23(28.40)$ & $60(54.55)^{\star}$ & $10(38.46)$ & $3(13.64)^{\star \#}$ & & \\
\hline Circumscribed & $5(6.17)$ & $12(10.91)$ & $3(11.54)$ & $8(36.36)$ & & \\
\hline Angular & $16(19.75)$ & $8(7.27)$ & $2(7.69)$ & $3(13.64)$ & & \\
\hline Spiculated & $28(34.57)$ & $21(19.09)$ & $8(30.77)$ & $2(9.09)$ & & \\
\hline Microlobulated & $9(11.11)$ & $9(8.18)$ & $3(11.54)$ & $6(27.27)$ & & \\
\hline BI-RADS category: & & & & & 5.440 & 0.142 \\
\hline $4 a$ & $6(7.41)$ & $4(3.64)$ & $2(7.69)$ & $2(9.09)$ & & \\
\hline $4 \mathrm{~b}$ & $25(30.86)$ & $23(20.91)$ & $7(26.92)$ & $4(18.19)$ & & \\
\hline $4 c$ & $29(35.80)$ & $40(36.36)$ & $8(30.77)$ & $8(36.36)$ & & \\
\hline 5 & $21(25.93)$ & $43(39.09)$ & $9(34.62)$ & $8(36.36)$ & & \\
\hline
\end{tabular}

Compared with $L A$ * represents $p<0.05$; Compared with $L B{ }^{*}$ represents $p<0.05$; Compared with HER2+ ${ }^{\#}$ represents $p<0.05$. LA - Luminal A, LB - Luminal B, HER2+- human epidermal growth factor receptor 2-positive, TN - triple-negative, BI-RADS - Breast Imaging Reporting and Data System. 
Table II. Microvascularity and tissue stiffness characteristics among the four molecular subtypes of invasive ductal carcinoma

\begin{tabular}{|c|c|c|c|c|c|c|}
\hline Variables & $\mathrm{LA}(n=81)$ & LB $(n=110)$ & HER $2+(n=26)$ & $\mathrm{TN}(n=22)$ & $\begin{array}{l}\chi^{2} / F \\
\text { value }\end{array}$ & $P$-value \\
\hline $\begin{array}{l}\text { Adler's } \\
\text { classification: }\end{array}$ & & & & & 7.280 & 0.064 \\
\hline 0 & $9(11.11)$ & $3(2.75)$ & $2(7.69)$ & $3(13.63)$ & & \\
\hline 1 & $25(30.87)$ & $42(38.54)$ & $4(15.38)$ & $7(31.82)$ & & \\
\hline 2 & $25(30.86)$ & $41(37.61)$ & $6(23.08)$ & $7(31.82)$ & & \\
\hline 3 & $22(27.16)$ & $23(21.10)$ & $14(53.85)$ & $5(22.73)$ & & \\
\hline SMI pixels & $1158(437-2400)$ & 981 (392-2168) & $3077.5(754-5668)$ & $622.5(224-3297)$ & 8.081 & 0.044 \\
\hline ROI pixels & $\begin{array}{c}44632 \\
(31227-69216)\end{array}$ & $\begin{array}{c}41611 \\
(24255-81060)\end{array}$ & $\begin{array}{c}49235 \\
(22191-74629)\end{array}$ & $\begin{array}{c}43801 \\
(20781-70490)\end{array}$ & 0.777 & 0.855 \\
\hline $\mathrm{SMI}$ area $\left[\mathrm{cm}^{2}\right]$ & $0.05(0.02-0.11)$ & $0.04(0.02-0.08)$ & $0.15(0.03-0.29)$ & $0.04(0.01-0.14)$ & 8.516 & 0.037 \\
\hline $\mathrm{ROI}$ area $\left[\mathrm{cm}^{2}\right]$ & $1.90(1.22-3.57)$ & $1.64(1.00-3.75)$ & $2.51(0.83-5.32)$ & $1.83(0.84-3.91)$ & 1.143 & 0.767 \\
\hline Ratio (\%) & $2.8(1.3-5.2)$ & $2.2(1.0-4.3)$ & $5.2(2.5-10.6)^{\#}$ & $2.2(1.1-5.3)$ & 12.668 & 0.005 \\
\hline Ave.T1 [kPa] & $94.7(57.2-121.2)$ & $109.1(72.0-147.1)$ & $127.5(66.8-154.8)$ & $125.7(65.5-149.4)$ & 9.213 & 0.027 \\
\hline SD.T1 [kPa] & $12.1(7.7-21.1)$ & $12.6(8.4-18.1)$ & $11.0(5.0-16.2)$ & $11.0(6.9-19.8)$ & 2.120 & 0.548 \\
\hline Ave.T2 [kPa] & $45.7(32.7-61.9)$ & $44.2(24.9-63.2)$ & $44.9(36.4-63.3)$ & $56.3(41.0-70.4)$ & 3.174 & 0.366 \\
\hline SD.T2 [kPa] & $24.4(17.1-36.2)$ & $26.8(15.3-37.8)$ & $29.3(14.1-39.4)$ & $33.8(13.6-41.7)$ & 2.175 & 0.537 \\
\hline Ave. $\mathrm{R}[\mathrm{kPa}]$ & $15.1(9.2-23.9)$ & $11.2(7.2-18.0)$ & $9.3(7.0-22.1)$ & $10.8(9.8-24.1)$ & 8.313 & 0.040 \\
\hline SD.R [kPa] & $2.3(1.1-3.9)$ & $1.6(0.6-2.7)$ & $1.3(0.6-2.3)$ & $1.5(0.8-3.6)$ & 12.187 & 0.007 \\
\hline Ratio1 [kPa] & $5.35(2.94-9.70)$ & $7.64(4.30-14.64)$ & $8.53(4.97-19.98)$ & $8.71(2.91-14.18)$ & 10.834 & 0.013 \\
\hline Ratio2 [kPa] & $2.86(1.68-4.32)$ & $3.41(2.07-5.78)$ & $3.99(2.20-7.84)$ & $3.39(2.04-6.90)$ & 6.351 & 0.096 \\
\hline
\end{tabular}

and LB ( $p=0.00182)$, with statistically significant differences. The percentages of patients with lymph node metastasis were $23.08 \%$ in $\mathrm{HER} 2+$, $27.27 \%$ in TNBC, $35.45 \%$ in LB, and $37.04 \%$ in LA with $p=0.519$ (Table I).

\section{Sonographic characteristics among the four molecular subtypes of IDC}

There were no statistically significant differences in the incidence of breast cancer on the left or right, tumour size, and maximum depth among the four subtypes. The shapes of LA (92.59\%), LB (89.09\%), HER2+ (92.31\%), and TNBC (81.82\%) subtypes tended to be irregular, while round or oval shapes were less common. Regarding orientation, tumour growth parallel to the skin was more common in the four subtypes of IDC and LA tended to be not parallel compared to HER2+ $(p=$ $0.0144)$. The echo pattern of tumours is the most basic ultrasonic characteristic, and there was an obvious difference among the four subtypes ( $p<$ 0.001). Hypoechoic pattern was more common in all four subtypes compared to complex cystic and solid pattern and LA was less common than LB in hypoechoic $(p=0.0006)$. As for the posterior features, there were significant differences among the four subtypes ( $p=0.019$ ). 55.55\% and 39.09\% of the LA and LB cases, respectively, had posterior acoustic shadowing compared with $23.08 \%$ of HER2+ cases and $18.18 \%$ of TNBC cases. No posterior features was more common in the TNBC subtype than in LA $(p=0.015)$. As for calcification, there was a statistically significant difference in calcifications among the four subtypes ( $p=$ 0.005). LA and HER2+ were more associated with the presence of calcifications, while the absence of calcifications was more common in the TNBC subtype. The presence of calcifications in the LA subtype was more common than in the LB subtype $(p=0.0120)$. Tumour margins in the LA, LB, and HER2+ subtypes tended to be indistinct (28.40\%, $54.55 \%$, and $38.46 \%$, respectively) and spiculated $(34.57 \%, 19.09 \%$, and $30.77 \%$, respectively), while circumscribed, angular, and microlobulated margins were less common. Tumour margins in the TNBC subtype were more commonly circumscribed (36.36\%) and microlobulated (27.27\%). Meanwhile, indistinct in LB was more common than LA ( $p=0.0048)$, while indistinct in TNBC subtype was less common than LA $(p=0.0024)$ and 
Table III. Sonographic characteristics between two invasive ductal carcinoma LB subtypes

\begin{tabular}{|c|c|c|c|c|}
\hline Variables & LB $($ HER2-) $(n=82)$ & LB $($ HER $2+)(n=28)$ & $\chi^{2 / Z / t}$ value & $P$-value \\
\hline Position: & & & 0.000 & 0.994 \\
\hline Left & $44(53.66)$ & $15(53.57)$ & & \\
\hline Right & $38(46.34)$ & $13(46.43)$ & & \\
\hline Size $[\mathrm{mm}]$ & $19.4(14.3-28.7)$ & $21.0(15.8-30.8)$ & 0.662 & 0.508 \\
\hline Maximum depth [mm] & $19.3(16.5-22.9)$ & $22.3(18.0-25.6)$ & 1.383 & 0.167 \\
\hline Shape: & & & 0.000 & 1.000 \\
\hline Round/oval & $9(10.98)$ & $3(10.71)$ & & \\
\hline Irregular & $73(89.02)$ & $25(89.29)$ & & \\
\hline Orientation: & & & 1.314 & 0.252 \\
\hline Parallel & $55(67.07)$ & $22(78.57)$ & & \\
\hline Not parallel & $27(32.93)$ & $6(21.43)$ & & \\
\hline Echo pattern: & & & 0.028 & 0.867 \\
\hline Hypoechoic & $76(92.68)$ & $25(89.29)$ & & \\
\hline Complex cystic and solid & $6(7.32)$ & $3(10.71)$ & & \\
\hline Posterior features: & & & & 0.711 \\
\hline No posterior features & $25(30.49)$ & $9(32.14)$ & & \\
\hline Shadowing & $30(36.58)$ & $13(46.43)$ & & \\
\hline Enhancement & $23(28.05)$ & $5(17.86)$ & & \\
\hline Combined pattern & $4(4.88)$ & $1(3.57)$ & & \\
\hline Calcification: & & & 1.234 & 0.267 \\
\hline Present & $59(71.95)$ & $17(60.71)$ & & \\
\hline Absent & $23(28.05)$ & $11(39.29)$ & & \\
\hline \multicolumn{5}{|l|}{ Margin: } \\
\hline Indistinct & $49(59.76)$ & $11(39.29)$ & & 0.004 \\
\hline Circumscribed & $10(12.19)$ & $2(7.14)$ & & \\
\hline Angular & $7(8.54)$ & $1(3.57)$ & & \\
\hline Spiculated & $14(17.07)$ & $7(25.00)$ & & \\
\hline Microlobulated & $2(2.44)$ & $7(25.00)$ & & \\
\hline BI-RADS category: & & & 0.314 & 0.575 \\
\hline $4 a$ & $2(2.44)$ & $1(3.57)$ & & \\
\hline $4 \mathrm{~b}$ & 15 (18.29) & $2(7.14)$ & & \\
\hline $4 c$ & $32(39.03)$ & $18(64.29)$ & & \\
\hline 5 & $33(40.24)$ & $7(25)$ & & \\
\hline
\end{tabular}

$L B$ - Luminal B, HER2- - human epidermal growth factor receptor 2-negative, HER2+-human epidermal growth factor receptor 2-positive, BI-RADS - Breast Imaging Reporting and Data System.

LB $(p=0.000567924)$. The BI-RADS tumour classification had no significant variation among the four subtypes ( $p=0.142)$ (Table I).

\section{Microvascularity and tissue stiffness characteristics among the four molecular subtypes of IDC}

SMI pixels, SMI area, and the ratio of SMI pixels to ROI pixels among the four subtypes had statistically significant differences ( $p=0.044,0.037$, and 0.005 , respectively). The ratio of SMI pixels to ROI pixels of the HER2+ subtype was higher than that of the LB subtype ( $p=0.018)$. Adler's classifi- cation, $\mathrm{ROI}$ pixels, and ROI area were not different among the four subtypes, but there were differences in Ave.T1, Ave.R, SD.R, and Ratio1 ( $p=0.027$, $0.040,0.007$, and 0.013, respectively). SD.T1, Ave. T2, SD.T2, and Ratio2 were not significantly different among the subtypes (Table II).

Sonographic, microvascularity, and tissue stiffness characteristics between two IDC LB subtypes

We further analysed the differences in ultrasound, microvascularity, and tissue hardness between LB (HER2+) and LB (HER2-) subtypes. Nota- 
Table IV. Microvascularity and tissue stiffness characteristics between two invasive ductal carcinoma LB subtypes

\begin{tabular}{|c|c|c|c|c|}
\hline Variables & LB $($ HER2-) $(n=82)$ & LB $($ HER2 +$)(n=28)$ & $\chi^{2} / Z / t$ value & $P$-value \\
\hline Adler's classification: & & & -0.161 & 0.872 \\
\hline 0 & $2(2.44)$ & $1(3.70)$ & & \\
\hline 1 & $32(39.02)$ & $10(37.04)$ & & \\
\hline 2 & $30(36.59)$ & $11(40.74)$ & & \\
\hline 3 & $18(21.95)$ & $5(18.52)$ & & \\
\hline SMI pixels & $992(340-2168)$ & $933(471-2278)$ & 0.326 & 0.744 \\
\hline ROI pixels & $39983.5(23358-72062)$ & $46478(28165-94012)$ & 1.257 & 0.209 \\
\hline $\mathrm{SMI}$ area $\left[\mathrm{cm}^{2}\right]$ & $0.04(0.02-0.08)$ & $0.04(0.02-0.10)$ & 0.271 & 0.786 \\
\hline ROI area $\left[\mathrm{cm}^{2}\right]$ & $1.59(0.98-3.48)$ & $2.24(1.05-5.06)$ & 1.253 & 0.210 \\
\hline Ratio (\%) & $2.2(0.9-4.9)$ & $2.4(1.1-4.1)$ & -0.211 & 0.833 \\
\hline Ave.T1 [kPa] & $110.2(72.0-147.8)$ & $107(70.6-144.8)$ & -0.284 & 0.776 \\
\hline SD.T1 [kPa] & $13.7(8.4-20.2)$ & $11.4(8.4-14.7)$ & -1.109 & 0.267 \\
\hline Ave.T2 [kPa] & $44.5(30-63.5)$ & $44.2(23.4-63.2)$ & -0.312 & 0.755 \\
\hline SD.T2 [kPa] & $27.5(16.5-37.7)$ & $24.2(13.6-39)$ & -0.147 & 0.883 \\
\hline Ave. $\mathrm{R}[\mathrm{kPa}]$ & $11.5(7.2-18.3)$ & $10.5(6.7-14.7)$ & -0.856 & 0.392 \\
\hline SD.R [kPa] & $1.7(0.6-2.7)$ & $1.3(0.6-2.9)$ & -0.843 & 0.399 \\
\hline Ratio1 (\%) & $7.62(3.97-14.64)$ & $8.35(4.70-16.74)$ & 0.586 & 0.558 \\
\hline Ratio2 (\%) & $3.44(2.00-5.78)$ & $3.35(2.40-5.87)$ & 0.151 & 0.880 \\
\hline
\end{tabular}

LB - luminal B, HER2- - human epidermal growth factor receptor 2-negative, HER2+- human epidermal growth factor receptor 2-positive, $S M I$ - superb microvascular imaging, ROI - region of interest, Ratio - the ratio of SMI pixels to ROI pixels, Ave.T1 - the average of T1, SD.T1 - the standard deviation of T1, Ave.T2 - the average of T2, SD.T2 - the standard deviation of T2, Ave.R - the average of region R, $S D . R$ - the standard deviation of region R, Ratio 1 - the ratio of Ave.T1 to Ave.R, Ratio2 - the ratio of Ave.T2 to Ave.R.

bly, there was a statistically significant difference in the type of margins between the two groups ( $p=0.004)$. LB (HER2-) tended to be indistinct, spiculated, and circumscribed (59.76\%, 17.07\%, and $12.19 \%$, respectively), while LB (HER2+) tended to be indistinct, spiculated, and microlobulated (39.29\%, 25\%, and 25\%, respectively). There were no other statistically significant differences between the two groups (Tables III and IV).

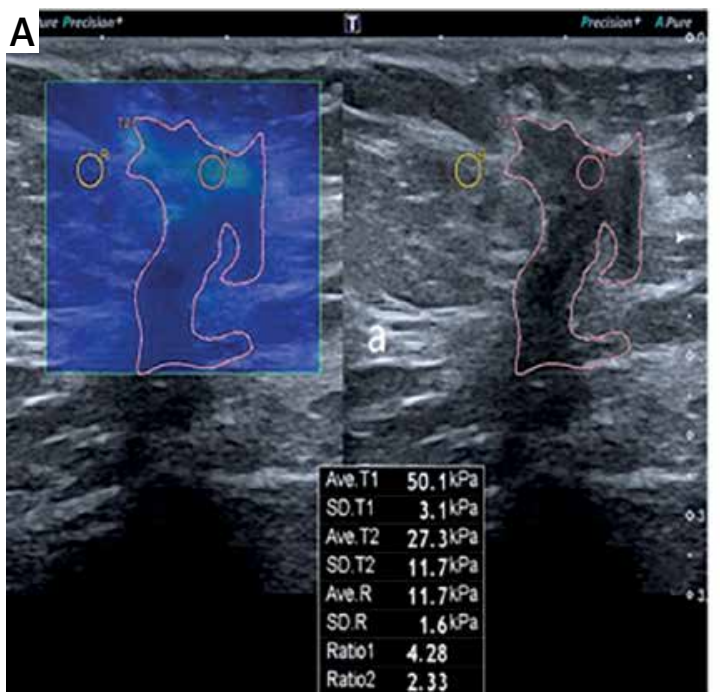

\section{Discussion}

The biological characteristics, treatments, and prognoses of different molecular subtypes of breast cancer are different $[2-4,17]$. Ultrasound is a preferred examination method for breast diseases [18-20]. The traditional Doppler method has the limitation of angle dependence and low signal-to-noise ratios and often fails to evaluate the flow of small vessels. A new Doppler ultra-

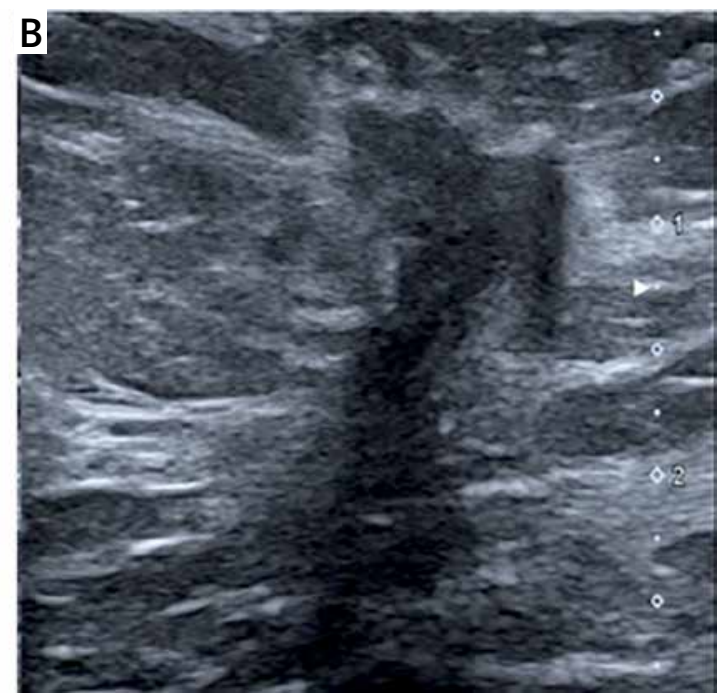

Figure 3. Ultrasonic manifestations of the Luminal A subtype. A - The shear-wave elastography image showed a relatively soft mass, the average of the stiffest part of the mass was $50.1 \mathrm{kPa}$, the average stiffness of the whole tumour was $27.3 \mathrm{kPa}$; B - B-mode image demonstrated a hypoechoic mass with spiculated margins and posterior acoustic shadowing 

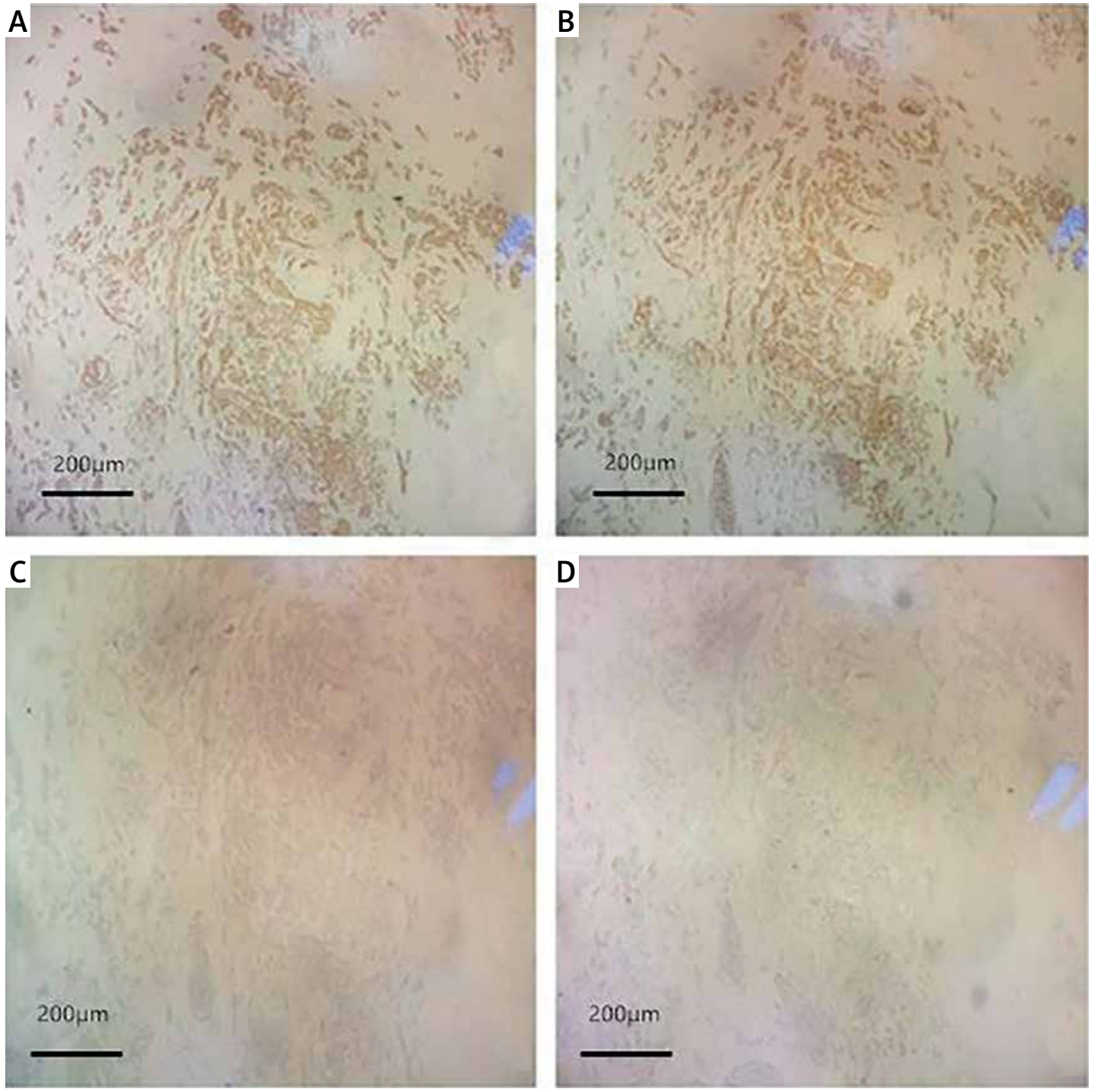

Figure 4. ER, PR, HER2+, and Ki-67 of Luminal A breast cancer are shown in A-D. Tumour cells stained dark brown are positive. In contrast, the unstained tumour cells are negative

sound technique, called SMI, can detect additional microvessel details, such as tiny branches and the distal parts of microvessels $[10,21]$. Tissue hardness is a characteristic of the breast evaluated by elasticity, an imaging method. This information can help distinguish benign from malignant breast tumours and improve patient management practices [22, 23]. Recently, a quantitative elastic measurement method called SWE using induced mechanical vibration that can locally quantify tissue hardness in kilopascals has been developed [11, 24-27]. To the best of our knowledge, there have been no reports of SMI, SWE, and BI-RADS lexicon in identifying the four subtypes of IDC.

The LA subtype of breast cancer has a relatively good prognosis [18]. Our results indicated that the LA subtype had the following characteristics: low histologic grade, posterior acoustic shadowing, spiculated margins, and relative softness (Figure 3). Immunohistochemical results of LA breast

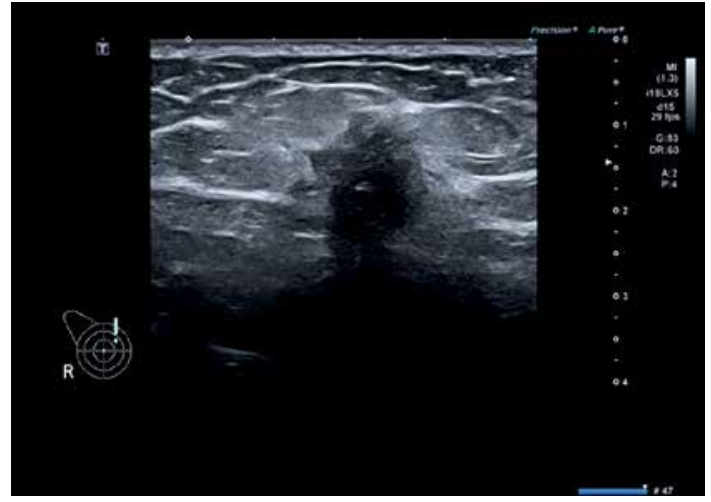

Figure 5. The main ultrasonographic manifestations of invasive ductal carcinoma in Luminal B subtype were indistinct margins and posterior acoustic shadowing

cancer are shown in Figure 4. Our study showed that most LA subtype cases had a low histologic grade, which was consistent with the results of 
previous studies $[18,28]$. It is generally believed that tumours have posterior echogenic shadowing due to excessive acoustic reflection or attenuation of tumour tissue compared to surrounding tissue and desmoplastic reactions, which are more likely to occur in low histologic grade tumours $[28,29]$. Previous studies have shown that low histological grade of ductal breast carcinoma is associated with ER+ or PR+ tumours and spiculated margins because of the presence of stromal responses representing spicules in low-grade tumours $[28,30]$. Ultrasound elastography can effectively distinguish benign from malignant breast tumours when combined with B-mode ultrasound [31]. Our study further explored the differences in hardness among the four IDC subtypes using quantitative SWE, and we found that the LA subtype was softer and had a better prognosis compared to the rest.

Conversely, the LB subtype had a relatively low prognosis, and was characterized with low histo-
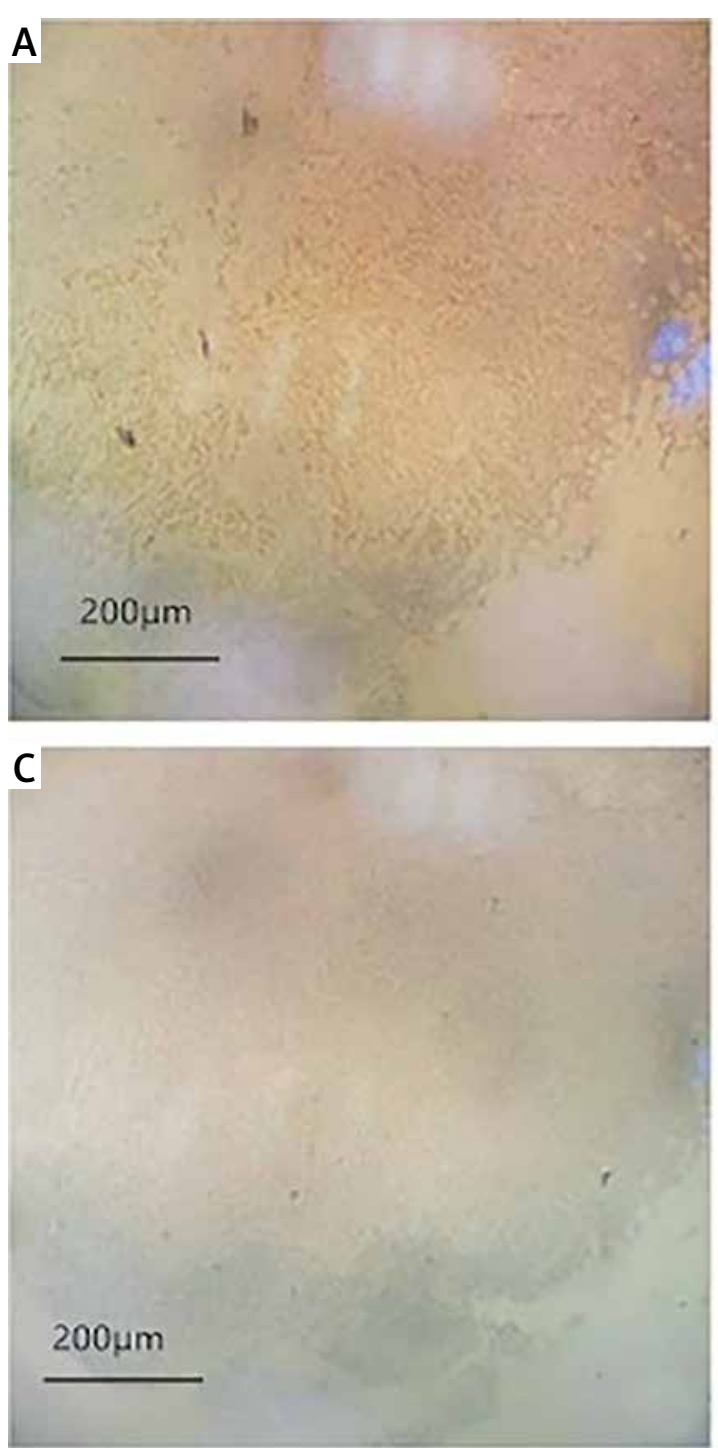

Figure 6. ER, PR, HER2+, and Ki-67 of LB (HER2-) breast cancer are shown in A-D. Tumour cells stained dark brown are positive. In contrast, the unstained tumour cells are negative
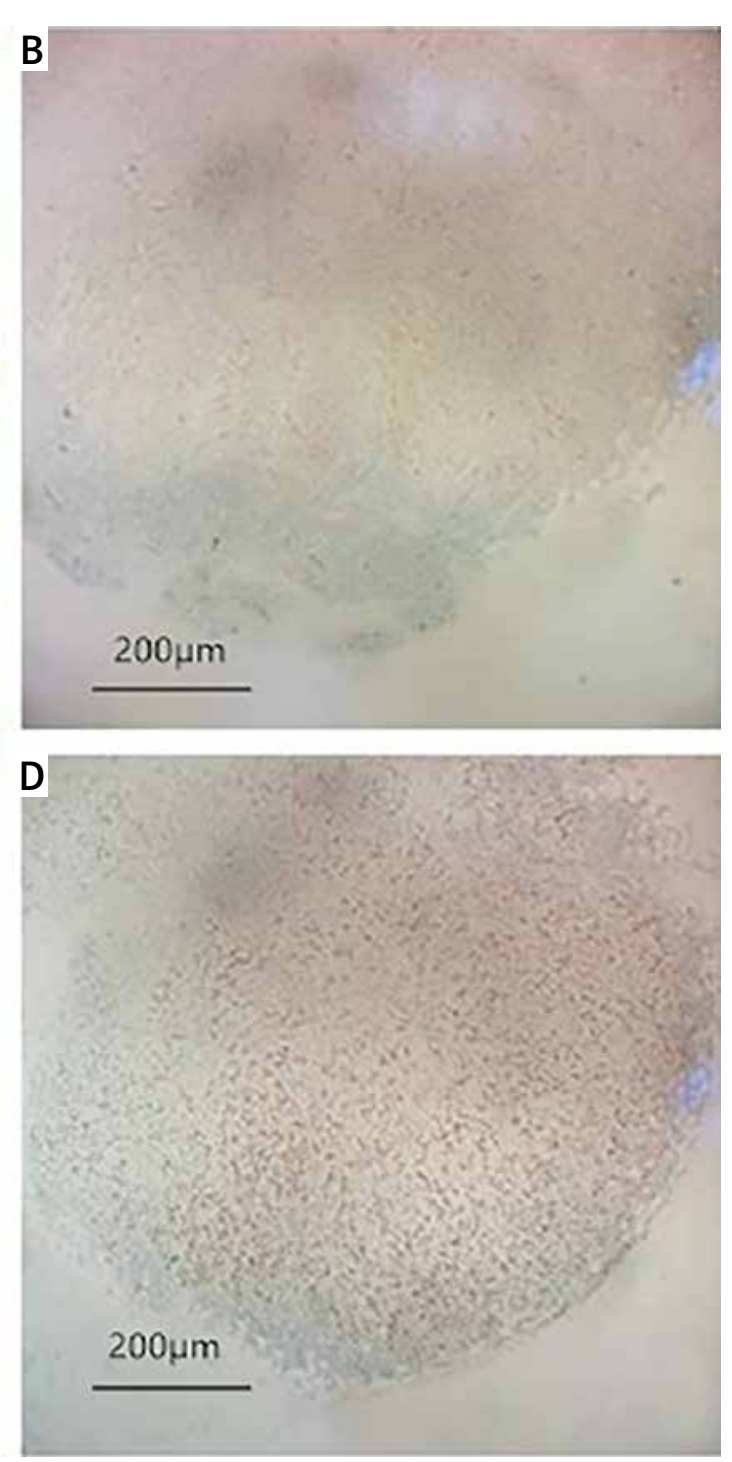

logical grade, posterior acoustic shadowing or no posterior features, and indistinct margins, which was consistent with previous studies [4, 17, 18] (Figure 5). Immunohistochemical results of LB (HER2-) and LB (HER2+) breast cancer are shown in Figures 6 and 7. In our study, the majority of the LB subtype cases had a low histological grade, which was different from a previous study [19]. The reason for this difference may be that the proportions of LB (HER2+) and LB (HER2-) cases were not the same and our study was limited to IDC. The LB (HER2-) subtype requires endocrine therapy and chemotherapy, while the LB (HER2+) subtype requires additional anti-HER2 therapy during clinical treatment. Therefore, it is necessary to differentiate the ultrasonic manifestations of the two LB molecular subtypes. Notably, the margins were significantly different between the LB (HER2-) and LB (HER2 + ) groups $(p=0.004)$. However, there were no significant differences in other 

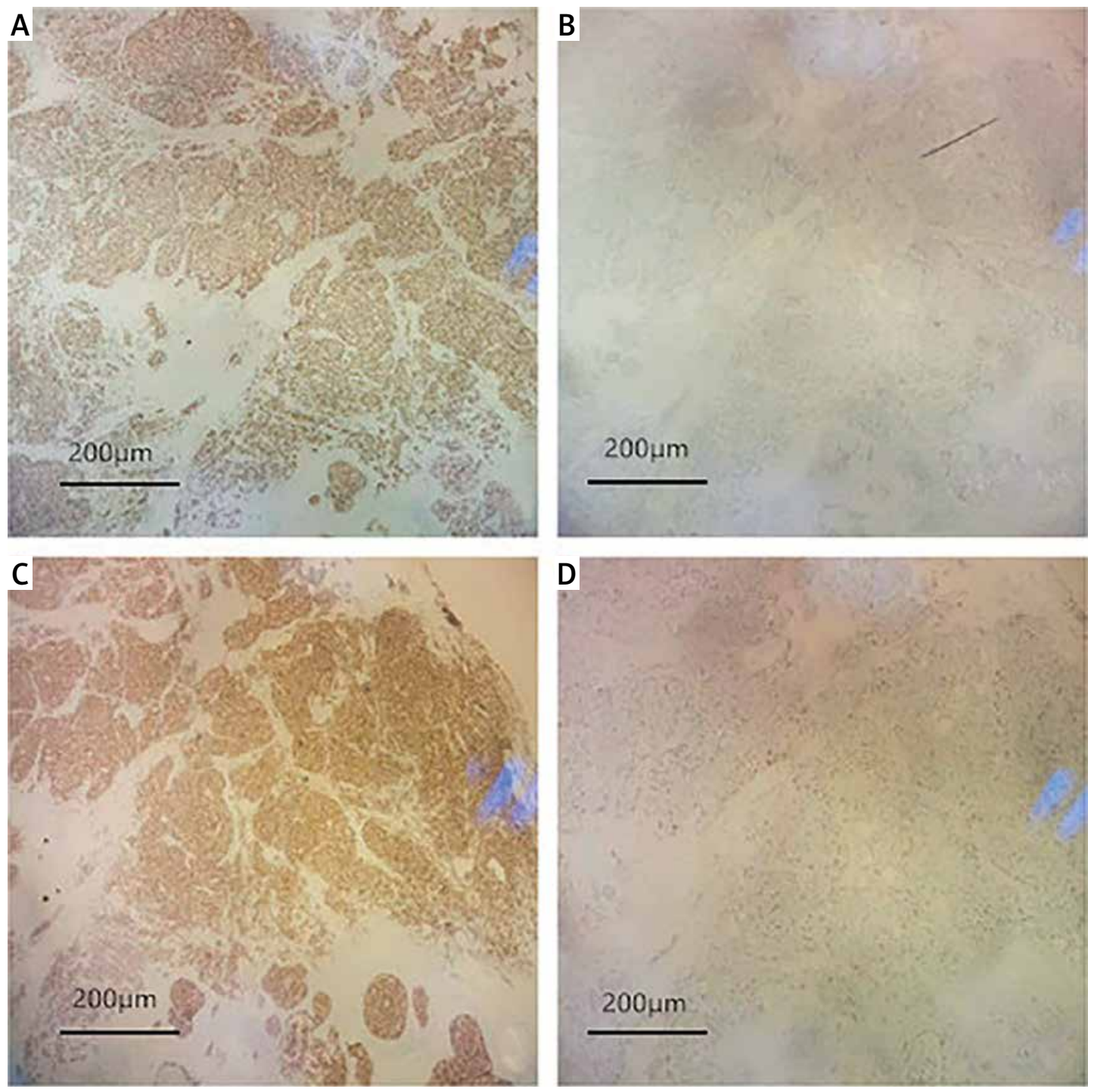

Figure 7. ER, PR, HER2+, and Ki-67 of LB (HER2+) breast cancer are shown in A-D. Tumour cells stained dark brown are positive. In contrast, the unstained tumour cells are negative

aspects between the two groups, which could be attributed to our small sample size or the actual absence of differences between the two subtypes. Therefore, further investigation of the differences between the two LB subtypes is needed.

HER2+ breast cancers were characterized by high histological grade, enhanced posterior acoustics or indifference, calcifications, spiculated or indistinct margins, vascularity, and being relatively stiff (Figure 8). Immunohistochemical results of HER2+ breast cancer are shown in Figure 9. The HER-2/neu gene plays an essential role in tumourigenicity, and its overexpression has been associated with poor prognostic indicators, such as high tumour grade, large tumour size, and negative hormone receptor status [32, 33]. Highly cellular and high-grade tumours tend to increase the spread of ultrasound and reduce ultrasonic attenuation compared to surrounding tissue; therefore, HER2+ tumours with a high grade may exhibit enhanced posterior acoustics [29, 30]. The expression of the HER2 oncogene was strongly associated with the occurrence of calcifications and spiculated margins [7, 34]. Indistinct margins and marked blood flow signals were associated with high histological grade [35]. Meanwhile, HER2 status is closely related to angiogenesis, which may be due to increased expression of vascular endothelial growth factor [36]. The increased stiffness and vascularity found in this subtype may be related to the poor prognosis of HER2+ breast cancer.

TNBCs are associated with invasive histological features, poor prognosis, no response to conventional endocrine therapy, and short survival time [37]. However, because TNBCs may appear benign, distinguishing them is important. Our study found that TNBCs are characterized by high histological grade, posterior echogenic enhancement, lack of calcifications, circumscribed or microlobulated margins, low blood flow signals, and stiff tissue 

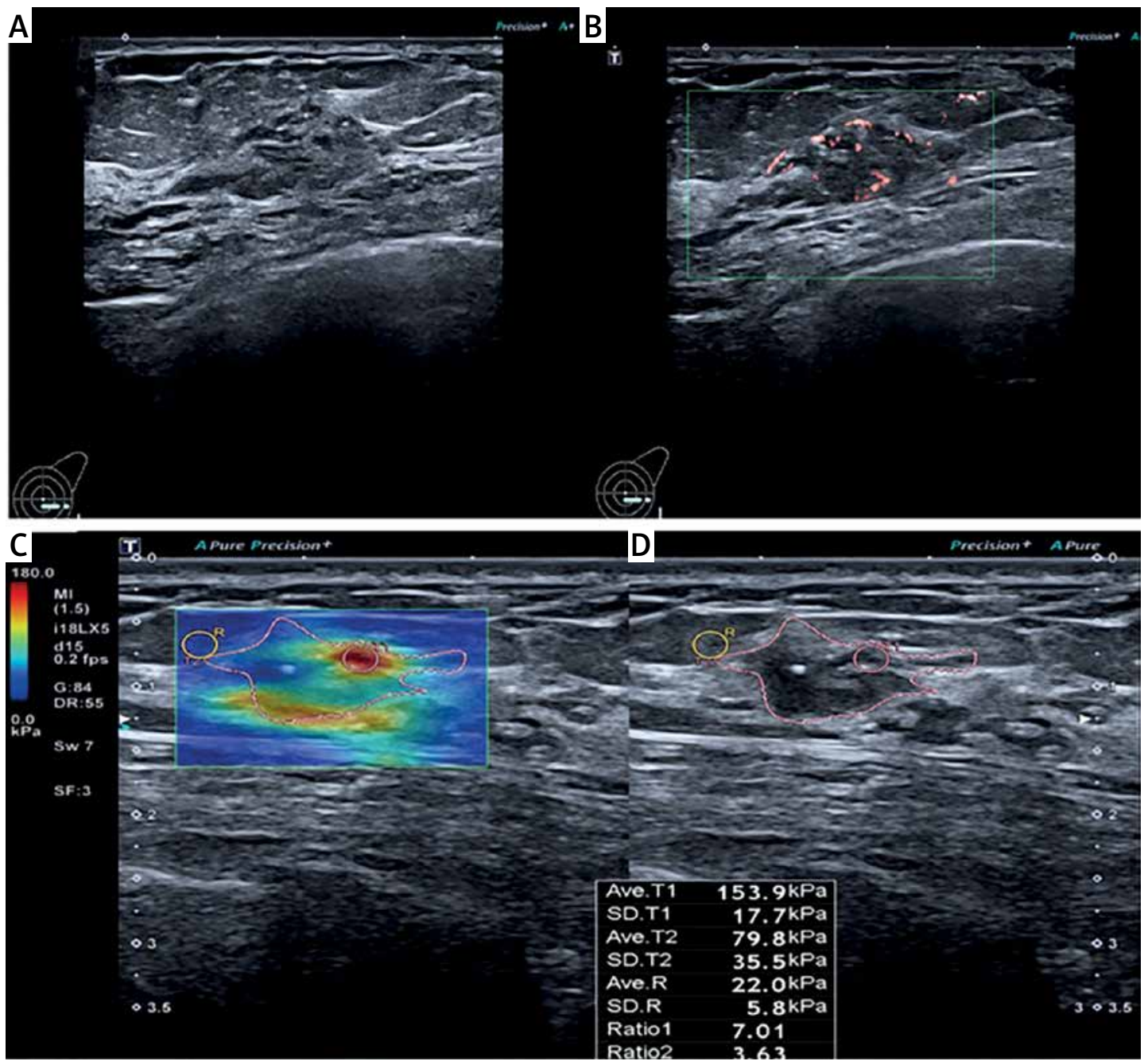

Figure 8. Ultrasonic manifestations of HER2+ subtype breast cancers. A, B - Two-dimensional ultrasound showed a mass with indistinct margins, calcifications, enhanced posterior acoustics and superb microvascular imaging showed the mass with marked blood flow signals (Adler grade 3). C, D - A mass with spiculated margins, calcifications and enhanced posterior acoustics, the shear-wave elastography image showed a relatively hard mass, the average of the stiffest part of the mass was $153.9 \mathrm{kPa}$, the average stiffness of the whole tumour was $79.8 \mathrm{kPa}$

(Figure 10). Immunohistochemical results of TNBC are shown in Figure 11. TNBC patients had more high-grade breast cancers than the other three subtypes [29]. Our study found that the majority of TNBCs presented with posterior echogenic enhancement, which may be due to the rapid growth of tumour patterns causing internal tissue necrosis, thus reducing the attenuation of ultrasound waves compared with the surrounding tissues [38]. Our study found that TNBCs are mainly associated with absence of calcifications, which is consistent with a previous study [15]. The circumscribed and microlobulated margins are associated not only with high histological grade and negative hormone receptor status but also with rapidly aggressive proliferating patterns in TNBCs, which have been described as pushing margins without an infiltrating stromal response [15, 35]. Our study showed that TNBCs were characterized by hypovascularity on SMI images, which was consistent with previous studies [18, 19]. We speculate that tumour necrosis leads to the destruction of tumour vascular components, resulting in a lack of vascularity. We also found that the tumour tissues of TNBCs were hard, which means that TNBC is more aggressive and tends to infiltrate surrounding tissues.

Our study had some limitations. First, its retrospective design meant that only patients with clinicopathological and ultrasonic data could be included. Second, the number of cases we studied was insufficient for the study of ultrasound and clinicopathological characteristics among the four subtypes. Third, we were not able to detect subtle differences between the two LB subtypes.

In conclusion, we used SMI, which can detect subtle blood flow, and SWE, which can quantify tissue hardness, to demonstrate differences in the sonographic and clinicopathological characteristics of the four IDC molecular subtypes. These 

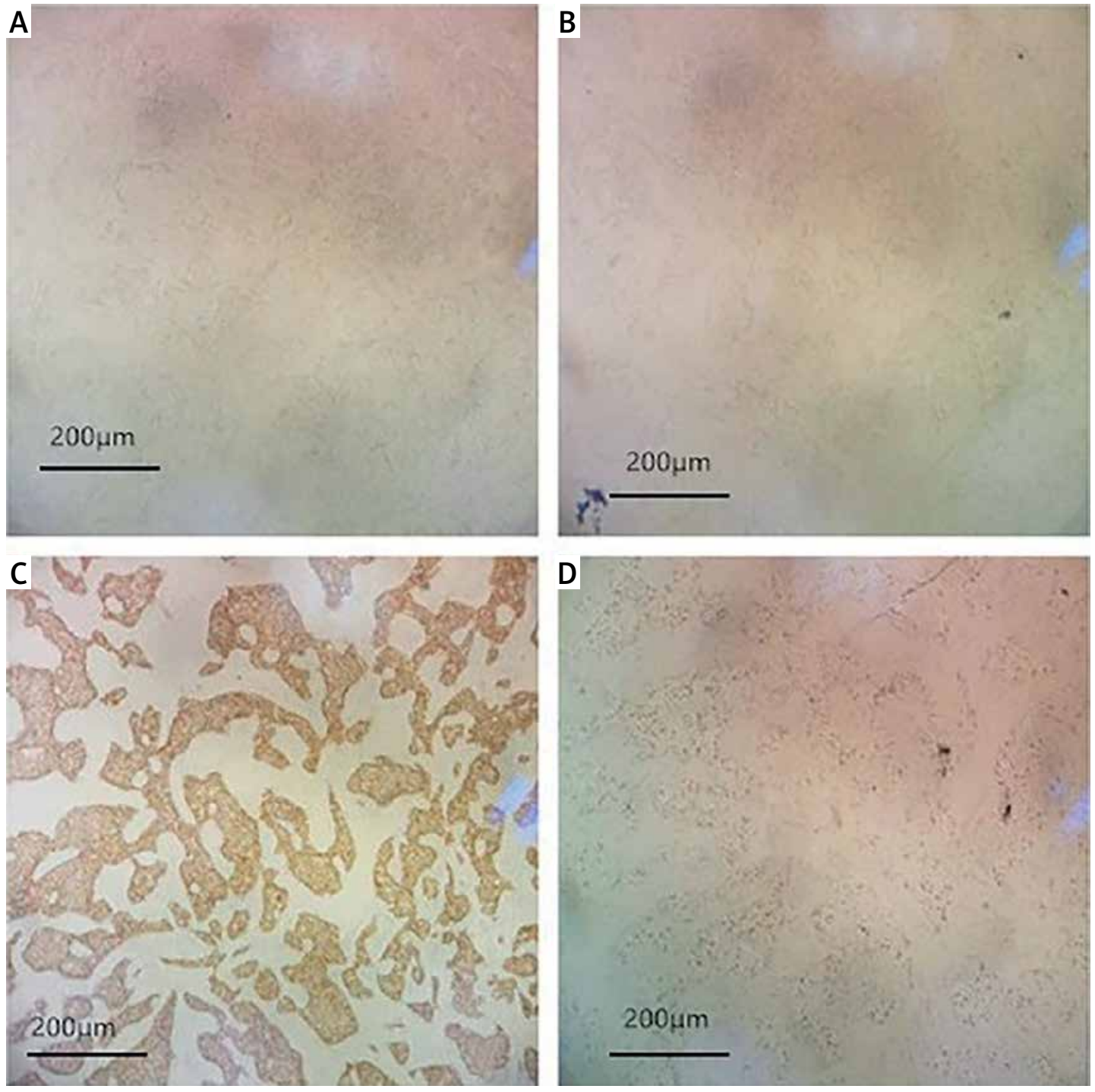

Figure 9. ER, PR, HER2+, and Ki-67 of HER2+ breast cancer are shown in A, B, C and D. Tumour cells stained dark brown are positive. In contrast, the unstained tumour cells are negative

findings, when applied in the appropriate clinical contexts, can help in the early diagnosis, selection of appropriate treatment methods, and prognosis prediction.

\section{Acknowledgments}

This study received funding from HAI YAN Science Foundation of Harbin Medical University Cancer Hospital (grant no. JJQN2021-12) and HAI YAN Science Foundation of Harbin Medical University Cancer Hospital (grant no. JJMS2020-01).

\section{Conflict of interest}

The authors declare no conflict of interest. 

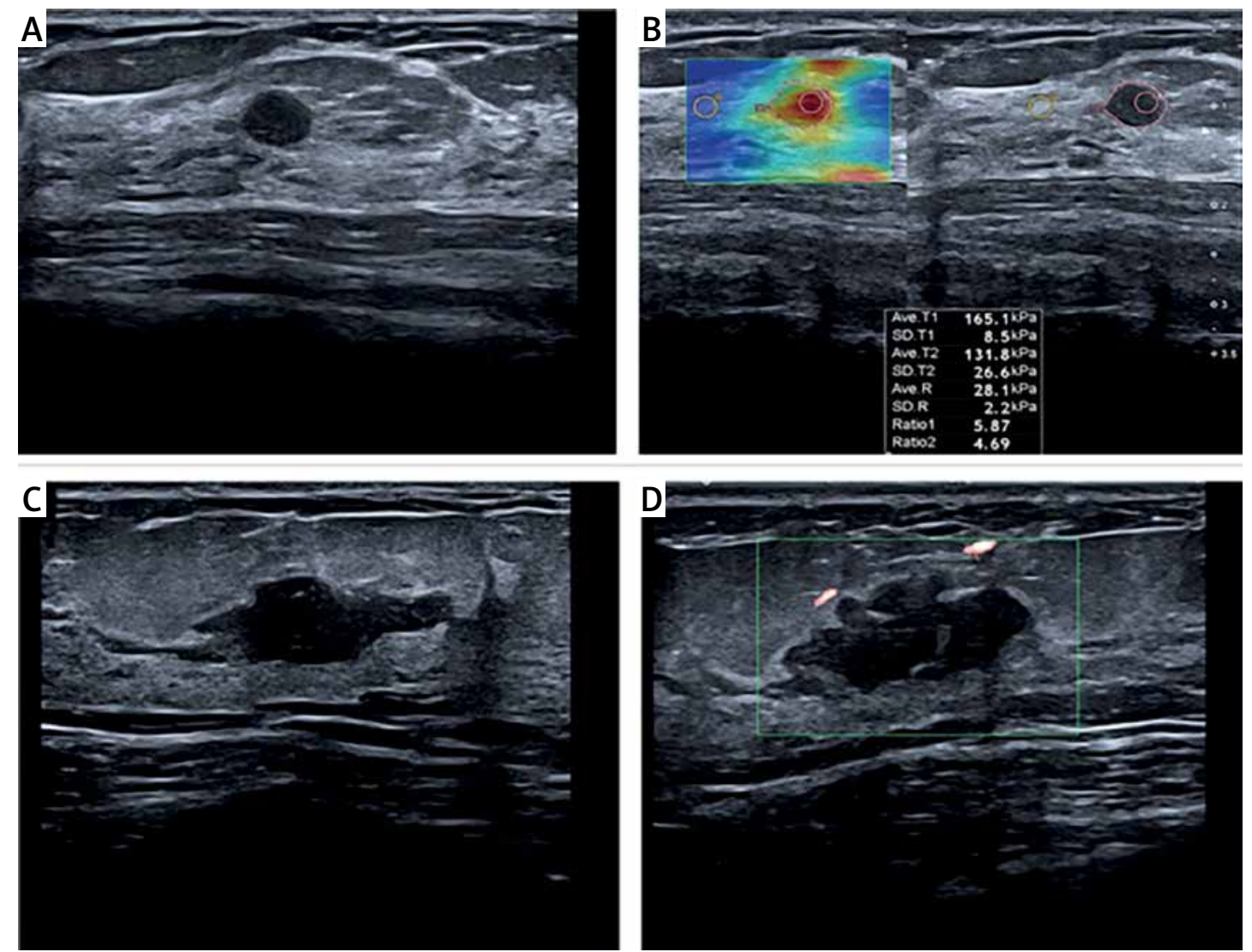

Figure 10. Triple negative breast cancers on ultrasound. A, B-Posterior echogenic enhancement, absence of calcifications, circumscribed margins and hard tissue. The average of the stiffest part of the mass was $165.1 \mathrm{kPa}$, the average stiffness of the whole tumour was $131.8 \mathrm{kPa}$. C, D - A hypoechoic mass of microlobulated margins with no calcifications and few blood flow signals on the superb microvascular imaging (Adler grade 1) 

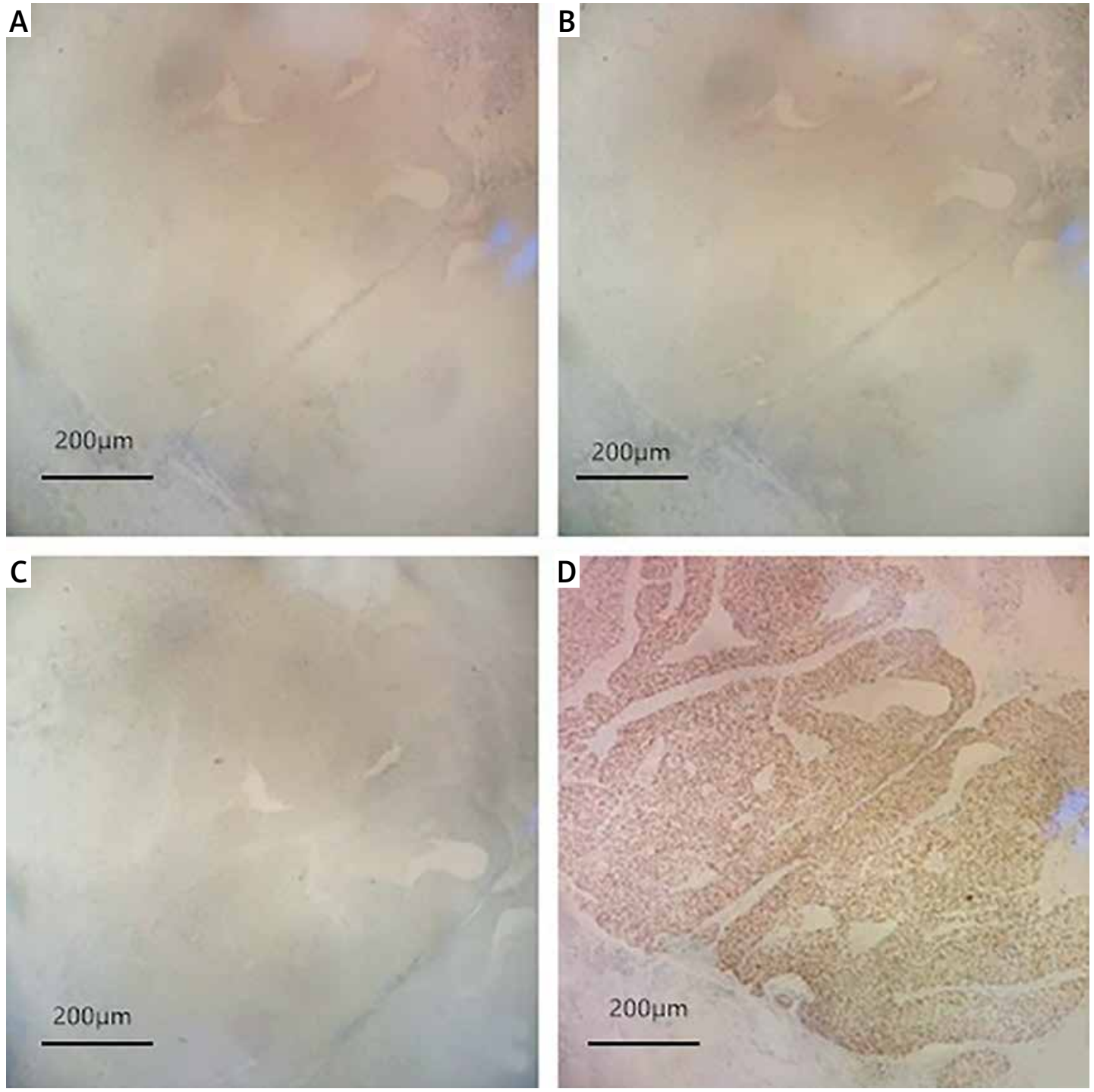

Figure 11. ER, PR, HER2+, and Ki-67 of TNBC are shown in A-D. The unstained tumour cells are negative

\section{References}

1. Li T, Mello-Thoms C, Brennan PC. Descriptive epidemiology of breast cancer in China: incidence, mortality, survival and prevalence. Breast Cancer Res Treat 2016; 159: 395-406.

2. Goldhirsch A, Winer EP, Coates AS, et al. Personalizing the treatment of women with early breast cancer: highlights of the St Gallen International Expert Consensus on the Primary Therapy of Early Breast Cancer 2013. Ann Oncol 2013; 24: 2206-23.

3. Goldhirsch A, Wood WC, Coates AS, et al. Strategies for subtypes--dealing with the diversity of breast cancer: highlights of the St. Gallen International Expert Consensus on the Primary Therapy of Early Breast Cancer 2011. Ann Oncol 2011; 22: 1736-47.

4. Wang GS, Zhu H, Bi SJ. Pathological features and prognosis of different molecular subtypes of breast cancer. Mol Med Rep 2012; 6: 779-82.

5. Kim SH, Seo BK, Lee J, et al. Correlation of ultrasound findings with histology, tumour grade, and biological markers in breast cancer. Acta Oncol 2008; 47: 1531-8.

6. Stavros AT, Thickman D, Rapp CL, Dennis MA, Parker SH, Sisney GA. Solid breast nodules: use of sonography to distinguish between benign and malignant lesions. Radiology 1995; 196: 123-34.

7. Wang Y, Ikeda DM, Narasimhan B, et al. Estrogen receptor-negative invasive breast cancer: imaging features of tumours with and without human epidermal growth factor receptor type 2 overexpression. Radiology 2008; 246: 367-75.

8. Bakdik S, Arslan S, Oncu F, et al. Effectiveness of superb microvascular Imaging for the differentiation of intraductal breast lesions. Med Ultrasonography 2018; 20: 306-12.

9. Artul S, Nseir W, Armaly Z, Soudack M. Superb microvascular imaging: added value and novel applications. J Clin Imaging Sci 2017; 7: 45.

10. Evans A, Whelehan P, Thomson K, et al. Quantitative shear wave ultrasound elastography: initial experience in solid breast masses. Breast Cancer Res 2010; 12: R104.

11. Lee SH, Chang JM, Han W, et al. Shear-wave elastography for the detection of residual breast cancer after neoadjuvant chemotherapy. Ann Surg Oncol 2015; 22: S376-84. 
12. Mercado LC. BI-RADS Update. Radiol Clin North Am 2014; 52: 481-7.

13. Bloom HJ, Richardson WW. Histological grading and prognosis in breast cancer; a study of 1409 cases of which 359 have been followed for 15 years. Br J Cancer 1957; 11: 359-77.

14. Prat A, Cheang MC, Martin M, et al. Prognostic significance of progesterone receptor-positive tumour cells within immunohistochemically defined luminal A breast cancer. J Clin Oncol 2013; 31: 203-9.

15. Yang WT, Dryden M, Broglio K, et al. Mammographic features of triple receptor-negative primary breast cancers in young premenopausal women. Breast Cancer Res Treat 2008; 111: 405-10.

16. Wolff AC, Hammond ME, Schwartz JN, et al. American Society of Clinical Oncology/College of American Pathologists guideline recommendations for human epidermal growth factor receptor 2 testing in breast cancer. J Clin Oncol 2007; 25: 118-45.

17. Turkoz FP, Solak M, Petekkaya I, et al. Association between common risk factors and molecular subtypes in breast cancer patients. Breast 2013; 22: 344-50.

18. Wu T, Li J, Wang D, et al. Identification of a correlation between the sonographic appearance and molecular subtype of invasive breast cancer: a review of 311 cases. Clin Imaging 2019; 53: 179-85.

19. Zhang L, Li J, Xiao Y, et al. Identifying ultrasound and clinical features of breast cancer molecular subtypes by ensemble decision. Sci Rep 2015; 5: 11085.

20. Man C, Wei ZW, Wang WP. Cystic breast lesions by conventional ultrasonography: sonographic subtype-pathologic correlation and BI-RADS assessment. Arch Med Sci 2014; 10: 76-83.

21. Yongfeng Z, Ping Z, Wengang L, Yang S, Shuangming T. Application of a novel microvascular imaging technique in breast lesion evaluation. Ultrasound Med Biol 2016; 42: 2097-105

22. Burnside ES, Hall TJ, Sommer AM, et al. Differentiating benign from malignant solid breast masses with US strain imaging. Radiology 2007; 245: 401-10.

23. Regner DM, Hesley GK, Hangiandreou NJ, et al. Breast lesions: evaluation with US strain imaging: clinical experience of multiple observers. Radiology 2006; 238 425-37.

24. Athanasiou A, Tardivon A, Tanter M, et al. Breast lesions: quantitative elastography with supersonic shear imaging: preliminary results. Radiology 2010; 256: 297-303.

25. Bercoff J, Tanter M, Fink $M$. Supersonic shear imaging: a new technique for soft tissue elasticity mapping. IEEE Trans Ultrason Ferroelectr Freq Control 2004; 51: 396 409.

26. Youk JH, Son EJ, Han K, Gweon HM, Kim JA. Performance of shear-wave elastography for breast masses using different region-of-interest (ROI) settings. Acta Radiol 2018; 59: 789-97.

27. Bae JS, Chang JM, Lee SH, Shin SU, Moon WK. Prediction of invasive breast cancer using shear-wave elastography in patients with biopsy-confirmed ductal carcinoma in situ. Eur Radiol 2017; 27: 7-15.

28. Zhu X, Ying J, Wang F, Wang J, Yang H. Estrogen receptor progesterone receptor, and human epidermal growth factor receptor 2 status in invasive breast cancer: a 3,198 cases study at National Cancer Center, China. Breast Cancer Res Treat 2014; 147: 551-5.

29. Thike AA, Cheok PY, Jara-Lazaro AR, Tan B, Tan P, Tan PH. Triple-negative breast cancer: clinicopathological char- acteristics and relationship with basal-like breast can cer. Mod Pathol 2010; 23: 123-33.

30. Lamb PM, Perry NM, Vinnicombe SJ, Wells CA. Correlation between ultrasound characteristics, mammographic findings and histological grade in patients with invasive ductal carcinoma of the breast. Clin Radiol 2000; 55: 40-4.

31. Chang JM, Won JK, Lee KB, Park IA, Yi A, Moon WK. Comparison of shear-wave and strain ultrasound elastography in the differentiation of benign and malignant breast lesions. Am J Roentgenol 2013; 201: W347-56.

32. Slamon DJ, Clark GM, Wong SG, Levin WJ, Ullrich A, McGuire WL. Human breast cancer: correlation of relapse and survival with amplification of the HER-2/neu oncogene. Science 1987; 235: 177-82.

33. Taucher S, Rudas M, Mader RM, et al. Do we need HER$2 /$ neu testing for all patients with primary breast carcinoma? Cancer 2003; 98: 2547-53.

34. Seo BK, Pisano ED, Kuzimak CM, et al. Correlation of HER-2/neu overexpression with mammography and age distribution in primary breast carcinomas. Acad Radiol 2006; 13: 1211-8.

35. Shin HJ, Kim HH, Huh MO, et al. Correlation between mammographic and sonographic findings and prognostic factors in patients with node-negative invasive breast cancer. Br J Radiol 2011; 84: 19-30.

36. Kumar R, Yarmand-Bagheri R. The role of HER2 in angiogenesis. Semin Oncol 2001; 28: 27-32.

37. Badowska-Kozakiewicz AM, Budzik MP, Liszcz A, et al. Clinicopathological factors associated with novel prognostic markers for patients with triple negative breast cancer. Arch Med Sci 2019; 15: 1433-42.

38. Lerma E, Barnadas A, Prat J. Triple negative breast carcinomas: similarities and differences with basal like carcinomas. Appl Immunohistochem Mol Morphol 2009; 17 483-94. 